\title{
A probabilistic model of geochemical rate distributions for reactive transport modelling
}

\author{
INNA KURGANSKAYA AND ANDREAS LUTTGE
}

University of Bremen

Presenting Author: inna.kurganskaya@uni-bremen.de

Material transport in geological environments involves geochemical reactions such as mineral dissolution, growth, and ion adsorption. The incorporation of reaction kinetics into reactive transport models is a fundamentally challenging problem due to the broad variance in intrinsic surface reactivity[1]. Variance of the "rate constant" or intrinsic rates typically used in reaction transport modelling substantially affects the outcome of the simulation. For example, Balashov et al.[2] revealed that the value of this constant substantially affects the rates of $\mathrm{CO}_{2}$ immobilization in greenhouse gas repositories. Lichtner and Tartakovsky[3] introduced a probability density function approach to treat the variance in rate constants caused by a variation in grain sizes. They found that the "effective" rate constant in this case has to be monitored and treated as a function over time. Fischer, Luttge, Arvidson and co-workers offered to consider "rate spectra" instead of rate constants for practical applications[4]. A milestone in the development of this concept is the implementation of the rate spectra concept into reactive transport codes. The major issue is the limited understanding of the statistical behavior and the scalability of rate spectra measured in experiments or obtained in Kinetic Monte Carlo (KMC) simulations[5]. In this study, we offer an alternative approach that is based on a probabilistic model of rate occurrence covering different space and time scales. The model is developed in connection with complementary $\mathrm{KMC}$ simulations allowing us to collect data sufficient for a rigorous statistical treatment. The resulting new modelling approach allows us to quantify the intrinsic rate variation. Our approach here is based on realistic physical processes that take place on the mineral surfaces, such as emergence and interaction of etch pits and atomic steps. The new model can be implemented into reactive transport codes and can be beneficial to the geochemical community and industry.

[1] Lüttge, Arvidson \& Fischer (2013) ELEMENTS, 183-8.

[2] Balashov, Guthrie, Hakala, Lopano, Rimstidt \& Brantley (2013) Applied Geochemistry, 41-56.

[3] Lichtner \& Tartakovsky (2003) Stochastic Environmental Research and Risk Assessment, 419-29.

[4] Fischer, Arvidson \& Lüttge (2012) Geochim. Cosmochim. Acta, 177-85.

[5] Fischer, Kurganskaya, Schäfer \& Lüttge (2014) Applied Geochemistry, 132-57. 